\title{
Effect of Unmodelled Actuator Dynamics on Feedback Linearised Systems and a Two Stage Feedback Linearisation Method
}

\author{
Mohamed Kara Mohamed and Alexander Lanzon
}

\begin{abstract}
This paper is concerned with the control design of nonlinear systems using feedback linearisation. The paper highlights the destabilisation effect of unmodelled actuator dynamics when applying feedback linearisation. To overcome this difficulty, a two stage feedback linearisation technique is proposed to compensate for actuator dynamics and subsequently linearise nonlinear systems. A case study of a tri-rotor UAV is used to showcase the benefits of the proposed method in comparison with classical feedback linearisation. The paper is written from a UAV application's point of view, however, the proposed procedure is still valid for any input-affine invertible nonlinear system.
\end{abstract}

\section{INTRODUCTION}

One of the common control design techniques for nonlinear systems is to linearise the system by cancelling the nonlinearity and then a linear control method can be used to synthesize a controller for the linearised model [5]. Cancelling the nonlinear term via feedback is known as feedback linearisation, and it can be either state-input feedback linearisation or input-output feedback linearisation. In this paper, we consider input-output feedback linearisation.

To simplify the implementation of feedback linearisation, several assumptions relating to the model of the nonlinear system and its operating point are considered. One of these assumptions, which is widely accepted in literature, is to neglect actuators dynamics. For instance, actuators dynamics are commonly neglected in Unmanned Aerial Vehicle (UAV) applications when synthesizing a controller (see for example [3], [2], [6] and the references therein). In these references, it is assumed that the actuators are fast and their dynamics can be safely neglected. In this work, we show that this assumption is not always valid and that when it comes to feedback linearisation, unmodeled actuators dynamics can have a vital destabilisation effect.

The effect of actuators dynamics on feedback linearisation has been considered by several research works, see for example [12], [1] and there references therein. However, the focus in these references is on how to recover the stability of the system when actuators dynamics are not available. In this work, we assume that actuators dynamics can be modelled and a two stage feedback linearisation method is then developed to handle actuators dynamics and linearise the nonlinear system. Although [4] tackles a similar problem, the work there is restricted to compensation of

Authors are with the Control Systems Centre, School of Electrical and Electronic Engineering, The University of Manchester, Manchester, UK, M13 9PL. Mohamed.KaraMohamed@Manchester.ac.uk, Alexander. Lanzon@Manchester.ac.uk actuators dynamics only for a specific SISO system. No such restrictions are made here.

The rest of the paper is organized as follows. In Section II, the input-output feedback linearisation technique is briefly reviewed. The undesirable effect of unmodelled actuators dynamics on input-output feedback linearisation is discussed in Section III. In Section IV, a two stage input-output feedback linearisation algorithm is proposed for a fully modelled system that includes actuators dynamics. A case study of a tri-rotor UAV is investigated in Section V and the summary of the paper is presented in Section VI.

\section{InPUt OUtPut FEEDBACK LinEARIZATION}

This section reviews the concept of input-output feedback linearisation [9] used to transform a nonlinear system into a linear one for a subsequent linear control design.

Consider a class of continuous multi-input multi-output (MIMO) fully actuated nonlinear systems of order $n$ and size $m \times m$ given by:

$$
\begin{aligned}
& \dot{x}=f(x)+\boldsymbol{G}(x) u \\
& y=h(x)
\end{aligned}
$$

Satisfying well known conditions, the nonlinear system (1) - (2) can be transformed into a linearised system of global normal form defined in a domain $\mathbb{D} \subset \mathbb{R}^{n}$ and given by:

$$
\begin{aligned}
& \dot{\zeta}=\boldsymbol{A}_{c} \zeta+\boldsymbol{B}_{c} \vartheta \\
& y=\boldsymbol{C}_{c} \zeta
\end{aligned}
$$

where $A_{c}, B_{c}$ and $C_{c}$ are block diagonal matrices of the Brunovsky canonical form. $\zeta=\left[\begin{array}{llll}\zeta_{1} & \zeta_{2} & \cdots & \zeta_{m}\end{array}\right]^{T}$ is defined by a new transformation mapping $\zeta=T(x)$ such that each element $\zeta_{i}$ is a vector of the output $y_{i}$ and its derivatives up to $y_{i}^{\left(r_{i}-1\right)}$ where $r_{i}$ is the relative degree of the output $y_{i}$. The feedback linearisation law from system (1) - (2) to the linearised system (3) - (4) is given by [9]:

$$
u(x, \vartheta)=\alpha(x)+\boldsymbol{\beta}(x)^{-1} \vartheta
$$

The matrix $\boldsymbol{\beta}(x)$ is called the decoupling matrix and it is assumed that this matrix is invertible in the domain $\mathbb{D}$. The new artificial input vector is defined as:

$$
\vartheta=\left[\begin{array}{llll}
y_{1}^{\left(r_{1}\right)} & y_{2}^{\left(r_{2}\right)} & \cdots & y_{m}^{\left(r_{m}\right)}
\end{array}\right]^{T} .
$$




\section{UNMODELLED ACTUATORS DYNAMiCS}

When applying feedback linearisation, actuators dynamics are usually neglected based on the assumption that actuators are fast enough to apply the required controller action without any considerable delay. In this section, we analyse the effect of unmodeled actuators dynamics on input-output feedback linearisation.

Consider a nonlinear system represented by (1) - (2) with actuators dynamics of order $n_{a}$ represented by:

$$
\begin{aligned}
\dot{x}_{a} & =\boldsymbol{A}_{a} x_{a}+\boldsymbol{B}_{a} u_{a} \\
y_{a} & =\boldsymbol{C}_{a} x_{a}
\end{aligned}
$$

We assume that the actuators system is asymptotically stable for all values $x_{a}(t) \in \mathbb{D}$. To impose actuators dynamics on the linearised system, we assume that the mapping $\zeta=T(x)$ is invertible. Rearranging the artificial control input $\vartheta$ to be in terms of the physical input $u$, the linearised system can be rewritten in terms of the physical input as:

$$
\begin{aligned}
& \dot{\zeta}=\boldsymbol{A}_{c} \zeta+\boldsymbol{B}_{c} \boldsymbol{\beta}\left(T^{-1}(\zeta)\right)\left(u-\alpha\left(T^{-1}(\zeta)\right)\right) \\
& y=\boldsymbol{C}_{c} \zeta
\end{aligned}
$$

The physical input to the system is now the output of the actuators $u=y_{a}=\boldsymbol{C}_{a} x_{a}$ and the feedback linearisation law is feeding to the actuators system $u_{a}=\alpha\left(T^{-1}(\zeta)\right)+$ $\boldsymbol{\beta}^{-1}\left(T^{-1}(\zeta)\right) \vartheta$ and therefore the system from $\vartheta$ to $y$ perturbed by actuators dynamics can be represented by:

$$
\begin{aligned}
\dot{\zeta} & =\boldsymbol{A}_{c} \zeta+\boldsymbol{B}_{c} \boldsymbol{\beta}\left(T^{-1}(\zeta)\right) \boldsymbol{C}_{a} x_{a}-\boldsymbol{B}_{c} \boldsymbol{\beta}\left(T^{-1}(\zeta)\right) \alpha\left(T^{-1}(\zeta)\right) \\
\dot{x}_{a} & =\boldsymbol{A}_{a} x_{a}+\boldsymbol{B}_{a} \alpha\left(T^{-1}(\zeta)\right)+\boldsymbol{B}_{a} \boldsymbol{\beta}^{-1}\left(T^{-1}(\zeta)\right) \vartheta \\
y & =\boldsymbol{C}_{c} \zeta
\end{aligned}
$$

The perturbed system in (10) - (12) is nonlinear and different from the linearised system (1) - (2). The order of this system from $\vartheta$ to $y$ is $n+n_{a}$. There is no guarantee that the synthesized controller for the linearised system in (3) - (4) will be able to stabilize the nonlinear system of higher order in (10) - (11). Moreover, if the controller manages to stabilize the nonlinear system, the performance will deteriorate. Therefore, actuators dynamics cannot be always neglected safely when using feedback linearisation as there is risk of instability.

\section{Two Stage FEedback Linearisation}

In this section, a two stage feedback linearisation technique is proposed to compensate for actuators dynamics and retain the validity of feedback linearisation of the nonlinear system. In this two stage method, the first stage handles actuators dynamics using inner loop linearisation/compensation and the second stage designs the outer loop by linearising the main nonlinear system. Figure 1 represents a block diagram of the proposed two stage feedback linearisation for nonlinear systems with actuators dynamics.

Consider a fully actuated nonlinear MIMO system given by (1) - (2) with actuators dynamics given by a nonlinear MIMO model of similar structure. The complete nonlinear

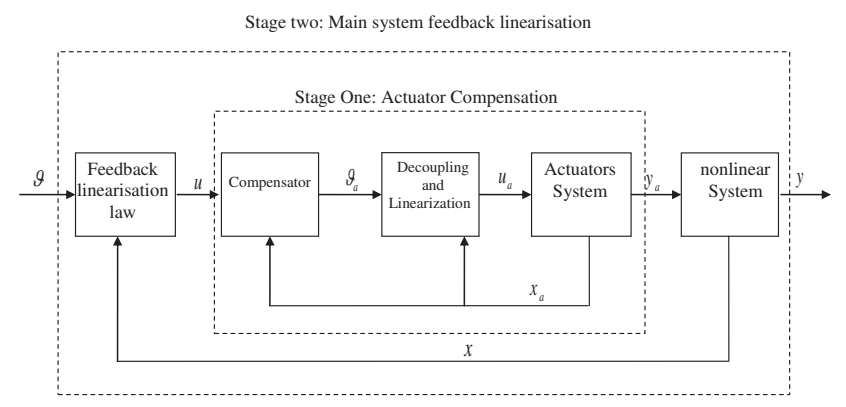

Fig. 1. Block diagram of the proposed two stage feedback linearisation.

system including actuators dynamics from $u_{a}$ to $y$ can be represented by:

$$
\begin{aligned}
& \dot{x}=f(x)+\boldsymbol{G}(x) h_{a}\left(x_{a}\right) \\
& \dot{x}_{a}=f_{a}\left(x_{a}\right)+\boldsymbol{G}_{a}\left(x_{a}\right) u_{a} \\
& y=h(x) .
\end{aligned}
$$

With the assumption that the actuators model satisfies the feedback linearisation conditions, the nonlinear actuators system can be linearised using input-output feedback linearisation to get:

$$
\begin{aligned}
\dot{\zeta}_{a} & =\boldsymbol{A}_{a} \zeta_{a}+\boldsymbol{B}_{a} \vartheta_{a} \\
y_{a} & =\boldsymbol{C}_{a} \zeta_{a},
\end{aligned}
$$

where $\boldsymbol{A}_{a}, \boldsymbol{B}_{a}, \boldsymbol{C}_{a}$ and $\vartheta_{a}$ are defined with respect to the actuators system. $\zeta_{a}=\left[\begin{array}{llll}\zeta_{a_{1}} & \zeta_{a_{2}} & \cdots & \zeta_{a_{m}}\end{array}\right]$ is a new actuators state vector defined by the mapping $\zeta_{a}=T_{a}\left(x_{a}\right)$ such that each element $\zeta_{a_{i}}$ is a vector of the output $y_{a_{i}}$ and its derivatives up to $y_{i}^{\left(r_{a_{i}}-1\right)}$ where $r_{a_{i}}$ is the relative degree of the output $y_{a_{i}}$. System (16) - (17) is a MIMO decoupled system in which each channel can be handled individually. For the i-th SISO channel of the linearised actuators system, let $\xi_{i}=\zeta_{a_{i}}$ with $\xi_{i}=\left[\begin{array}{llll}\xi_{i_{1}} & \xi_{i_{2}} & \cdots & \xi_{i_{r_{i}}}\end{array}\right]^{T}$, then the dynamics of the i-th linearised channel can be written as:

$$
\begin{aligned}
& \dot{\xi}_{i_{1}}=\xi_{i_{2}} \\
& \dot{\xi}_{i_{2}}=\xi_{i_{3}} \\
& \vdots \\
& \dot{\xi}_{i_{r_{i}}}=\vartheta_{a_{i}} \\
& y_{a_{i}}=\xi_{i_{1}} .
\end{aligned}
$$

The desire is to make the linearised actuators system follow a trajectory of reference signal input given by $u$. This reference input represents the feedback linearisation control law of the outer loop that will be discussed later. In this paper, backstepping control technique is used to achieve the input tracking and build the inner loop control law. Define the error system for the i-th actuators channel using the strict feedback 
structure as following:

$$
\begin{aligned}
e_{i_{1}} & =\xi_{i_{1}}-u_{i} \\
e_{i_{2}} & =\xi_{i_{2}}-z_{i_{1}}-\dot{u}_{i} \\
\vdots & \\
e_{i_{r_{a_{i}}}} & =\xi_{i_{r_{a_{i}}}}-z_{i_{\left(r_{a_{i}}-1\right)}}-u_{i}^{\left(r_{a_{i}}-1\right)},
\end{aligned}
$$

where $z_{i_{k}}, 1 \leq k \leq r_{a_{i}}$ is a backstepping control law that is used to achieve stability and convergence of the overall error system [10]. Using Lyapunov functions, $z_{i_{k}}$ can be designed as [10]:

$$
z_{i_{k}}=-e_{i_{k-1}}-c_{i_{k}} e_{i_{k}}+\dot{z}_{i_{k-1}}
$$

The final control law for the i-th channel can be represented as:

$$
\vartheta_{a_{i}}=z_{i_{a_{i}}}+u_{i}^{\left(r_{a_{i}}\right)}
$$

The resulting error system from (23) - (26) is given as:

$$
\left[\begin{array}{c}
\dot{e}_{i_{1}} \\
\dot{e}_{i_{2}} \\
\vdots \\
\dot{e}_{i_{r_{a_{i}}}}
\end{array}\right]=\left[\begin{array}{ccccc}
-c_{i_{1}} & 1 & 0 & \ldots & 0 \\
-1 & -c_{i_{2}} & 1 & \ldots & 0 \\
0 & -1 & \ddots & \ddots & \vdots \\
0 & 0 & \cdots & -1 & -c_{i_{r_{a_{i}}}}
\end{array}\right]\left[\begin{array}{c}
e_{i_{1}} \\
\dot{e}_{i_{2}} \\
\vdots \\
e_{i_{r_{a_{i}}}}
\end{array}\right]
$$

Using Lyapunov theory, it can be easily proved that the above system in (29) is globally uniformly asymptotically stable at the origin, which means that the global asymptotic tracking is achieved. The transient performance of the error system can be controlled by the design parameters $c_{i_{k}}$. In general, increasing $c_{i_{k}}$ will improve the transient performance of the error system [10]. This backstepping control design needs to be repeated for all actuators channels.

Upon completing the control design of the inner loop, one can put now $y_{a} \approx u$ assuming a high value of $c_{i_{k}}$ to achieve high bandwidth. Thereafter, the second stage can be implemented, which is input-output feedback linearisation of the main nonlinear system only bypassing the inner loop.

One point that might arise here is why the two stage feedback linearisation is needed given that the actuators dynamics are available and the whole system including actuators can be linearised in one step using the standard feedback linearisation procedure? The proposed two stage algorithm simplifies the implementation of feedback linearisation and is less conservative for the feedback linearisation law. For instance, consider a full nonlinear system including actuators dynamics as represented by (13) - (15). This system can be linearised as one system using standard feedback linearisation. However, this requires differentiation along both vector states $x_{a}$ and $x$, which means that the vector functions $f(\cdot), f_{a}(\cdot)$ and the column functions of $G(\cdot)$ and $G_{a}(\cdot)$ need to be smooth and differentiable up to the $n+n_{a}$ degree. This condition is more conservative and is not required in the two stage feedback linearisation as the actuators system and the plant are handled separately. A comparison study between the proposed two stage feedback linearisation and the standard linearisation on the whole system will be conducted when considering the example of the tri-rotor UAV in next section.

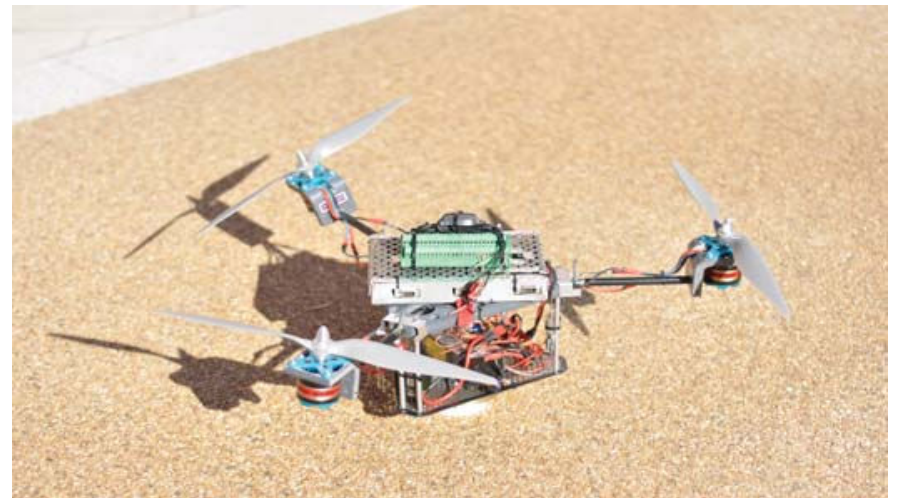

Fig. 2. The tri-rotor UAV system.

\section{Case Study: Design and Control of A TRI-ROTOR UAV}

This section is dedicated to the control design of a trirotor UAV as a case study of using the proposed two stage feedback linearisation to linearise a nonlinear system. The vehicle under consideration was proposed originally in [8] and is shown in Figure 2. The vehicle's dynamics expressed in the body coordinate system can be described by [8]:

$$
\begin{aligned}
& \dot{v}=g H_{g}-\boldsymbol{S}(\omega) v+\frac{k_{f}}{M_{t o t}} \boldsymbol{H}_{f} \rho \\
& \dot{\omega}=-(\boldsymbol{I})^{-1} \boldsymbol{S}(\omega) \boldsymbol{I} \omega+(\boldsymbol{I})^{-1}\left(k_{f} \boldsymbol{H}_{t}-k_{t} \boldsymbol{H}_{f}\right) \rho \\
& \dot{\eta}=\boldsymbol{\Psi} \omega \\
& \dot{\boldsymbol{\lambda}}=\boldsymbol{R}_{b}^{e} v
\end{aligned}
$$

Full definition of the notation and derivation of the model can be accessed in [7]. The output of the system is defined as the position and attitude of the vehicle, i.e., $y=\left[\begin{array}{ll}\eta & \lambda\end{array}\right]^{T}$.

In [8], an $\mathscr{H}_{\infty}$ controller was designed to stabilise the vehicle where input-output feedback linearisation is used to linearised the system. Actuators dynamics were neglected with the assumption that actuators are fast enough to apply the control action without any considerable delay. In this paper, this assumption is analysed and challenged, and then feedback linearisation for the full model that includes actuators dynamics is implemented. For convenience and comparative purposes, the controller designed in [8] is presented here again.

The case study includes 4 scenarios depending on the level of the UAV model and the implemented feedback linearisation as following:

Case 1: The system model does not include actuators dynamics, which is a replica of the case considered originally in [8].

Case 2: Actuators dynamics are included in the system model but not accounted for by the controller.

Case 3: The proposed two stage feedback linearisation is invoked to linearise the full system, i.e., including actuators dynamics.

Case 4: Classical feedback linearisation technique is used to linearise the full system. 
Cases 1 and 2 aim to demonstrate the undesirable effect of unmodelled actuators dynamics on feedback linearisation, while Cases 3 and 4 present a comparative study between the proposed two stage feedback linearisation and the classical feedback linearisation on the whole system. The performance of the controller in all cases is simulated using Matlab Simulink software. All simulations are considered for a scenario of horizontal hovering at height of $5 \mathrm{~m}$, i.e., the reference input is $(0,0,0)$ deg for the attitude and $(0,0,5)$ $\mathrm{m}$ for the position in the earth frame, and the vehicle was at a non-zero initial position and attitude.

1) Case 1: Control Synthesis without Actuators Dynamics: As mentioned earlier, this case represents the controller synthesised in [8] where no actuators dynamics are considered. In this case, the vector $\rho$ in (30) is considered as the the input vector of the system, i.e., $u=\rho$. The linearised plant is a double integrator representing single degree of freedom for translational and rotational motion and the feedback linearisation law is derived as:

$$
u=\boldsymbol{\beta}^{-1}\left(\vartheta-\left[\begin{array}{c}
\left(\dot{\boldsymbol{\Psi}}-\boldsymbol{\Psi} \boldsymbol{I}^{-1} \boldsymbol{S}(\omega) \boldsymbol{I}\right) \omega \\
g \boldsymbol{R}_{b}^{e} H_{g}
\end{array}\right]\right) .
$$

where $\vartheta$ by $y^{(2)}=\vartheta$ and the matrix $\boldsymbol{\beta}$ is defined as:

$$
\boldsymbol{\beta}=\left[\begin{array}{c}
\boldsymbol{\Psi} \boldsymbol{I}^{-1}\left(k_{f} H_{t}-k_{t} \boldsymbol{H}_{f}\right) \\
\frac{k_{f}}{M_{t o t}} \boldsymbol{R}_{b}^{e} \boldsymbol{H}_{f}
\end{array}\right] .
$$

It has been shown that $\operatorname{det}[\boldsymbol{\beta}()] \neq$.0 and the inverse $\boldsymbol{\beta}(.)^{-1}$ always exists for all states values of the system. Using the synthesised $\mathscr{H}_{\infty}$ LSDP controller in [8], the vehicle's position $\left(x_{v}, y_{v}, z_{v}\right)$ and attitude (pitch $\phi_{v}$, roll $\theta_{v}$, yaw $\left.\psi_{v}\right)$ related to the earth coordinate system and the control efforts in terms of the BLDC motors speeds $\omega_{m_{i}}$ and the tilting angles of the servo motors $\alpha_{s_{i}}$ are shown in Figures 3(a) and 3(b) respectively.

2) Case 2: Analysis of The Effect of Unmodeled Actuators Dynamics: In the previous case, actuators dynamics were neglected assuming that actuators are fast and their dynamics can be neglected. In this case, we challenge this assumption by investigating the effects of unmodeled actuators dynamics on the stability of the system.

The tri-rotor UAV has two types of actuators, BLDC motors and digital Servos. Neglecting the inductance effect, the dynamic model of the BLDC motors can be represented by a first order system [11]. Similarly, the servos combined with their drive circuits can be represented by first order systems using the supplied specifications of the servos step responses. Assuming identical BLDC motors and identical servo motors, and in state space form, the dynamics of the six actuators (three BLDC motors and three servo motors) can be written as:

$$
\begin{aligned}
& \dot{x}_{a}=\boldsymbol{A}_{a} x_{a}+\boldsymbol{B}_{a} u_{a} \\
& y_{a}=x_{a}
\end{aligned}
$$

where $\boldsymbol{A}_{a}$ and $\boldsymbol{B}_{a}$ are diagonal matrices with suitable dimension. $y_{a}$ is a vector of the rotational speeds of the BLDC
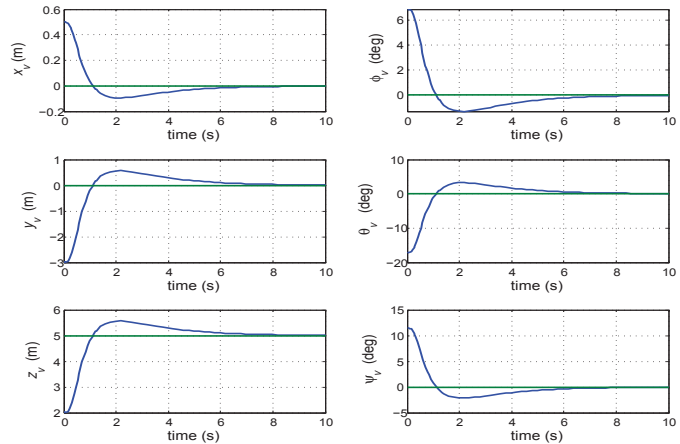

(a) The UAV position and attitude.
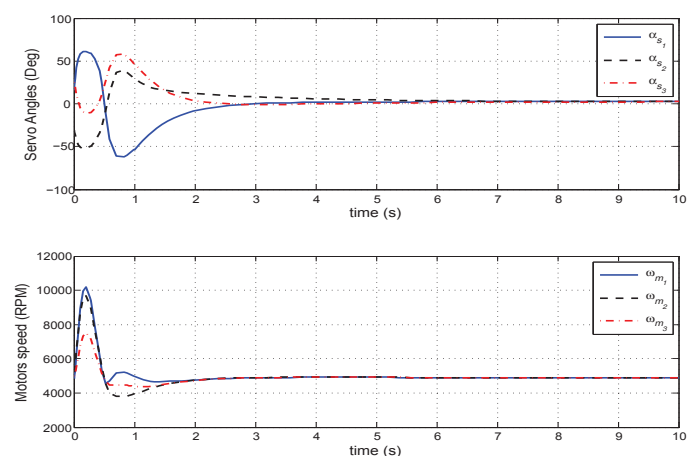

(b) The actuators performance.

Fig. 3. Case1: Control design for system without actuators dynamics [8].

motors and angles of the servo motors. $u_{a}$ is the driving voltages of the BDLC motors and the servo motors.

We now include the actuators dynamics in the simulation using the same controller considered in Case 1 to test the stability of the system. The effect of the unmodelled actuators dynamics on the performance of the UAV is reflected in Figure 4(a) with actuators effort shown in Figure 4(b). These figures clearly show that unmodelled actuators dynamics destabilize the UAV system. This observation is consistent with the result derived before in Section III.

3) Case 3: Two Stage Feedback Linearisation Technique: In this case, we invoke the two stage feedback linearisation technique developed in Section IV to linearise the system and then design an $\mathscr{H}_{\infty}$ controller. As the actuators system is a linear decoupled system, there is no need for a linearisation or decoupling process and the backstepping technique can be applied directly. The error system is defined as:

$$
e=y_{a}-y_{d}
$$

where $y_{d}=u$ and $u$ is the feedback linearisation law of the outer loop (to be designed later). This means:

$$
\begin{aligned}
\dot{e} & =\dot{y}_{a}-\dot{y}_{d} \\
& =\boldsymbol{A}_{a} x_{a}+\boldsymbol{B}_{a} u_{a}-\dot{y}_{d}
\end{aligned}
$$

We nominate the Lyapunov function $V=\frac{1}{2} e^{2}$, whose derivative is $\dot{V}=e\left(\boldsymbol{A}_{a} x_{a}+\boldsymbol{B}_{a} u_{a}-\dot{y}_{d}\right)$. To achieve global asymptotic 

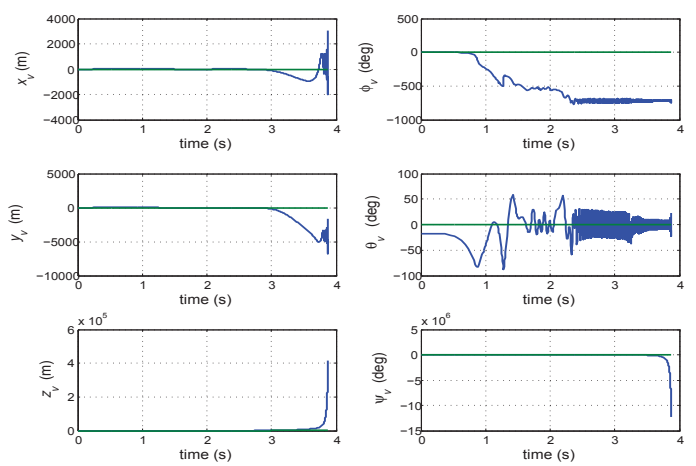

(a) The UAV position and attitude.
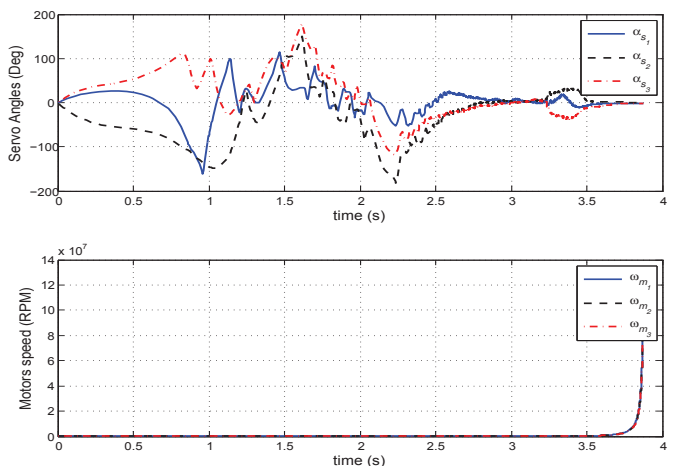

(b) The actuators performance.

Fig. 4. Case2: The undesirable destabilizing effect of unmodelled actuators dynamics.

stability at the origin, we design the control law, following Eq. (28), as:

$$
\begin{aligned}
u_{a} & =\boldsymbol{B}_{a}^{-1}\left[\dot{y}_{d}-\boldsymbol{A}_{a} x_{a}-\boldsymbol{c} e\right] \\
& =\boldsymbol{B}_{a}^{-1}\left[\dot{u}-\boldsymbol{A}_{a} x_{a}-\boldsymbol{c}\left(x_{a}-u\right)\right]
\end{aligned}
$$

where $c$ is a diagonal design matrix. To ensure high bandwidth and quick convergence of $y_{a}$ to $y_{d}$, we choose $c$ with large elements. Typically, $c_{i i}>100, i=1,2 \cdots, 6$, where $c_{i i}$ are the diagonal elements of the matrix $c$, are sufficient.

We now move to the second stage where we linearise the UAV system without the inner loop. This stage includes the linearisation of the UAV system as described in (30) (33). The feedback linearisation law is given in (34) and the resulting linearised system is a double integrator.

To demonstrate the system performance when using the two stage feedback linearisation, we implement the same controllers designed before in Case 1. In Figures 5(a) and 5(b) respectively, the system performance and the actuators efforts are depicted. It can be noted that Figure 5 is very similar to Figure 3 where actuators dynamics were not included. This is due to the inner stage of compensating and controlling actuators dynamics.

4) Case 4: Complete Model Classical Feedback Linearisation: For comparative study, classical feedback linearisation of the full system including actuators dynamics is implemented in this case. The study aims to clarify the benefits
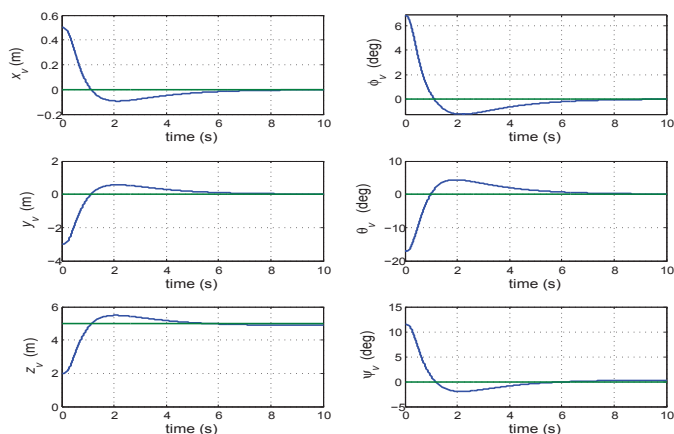

(a) The UAV position and attidute.
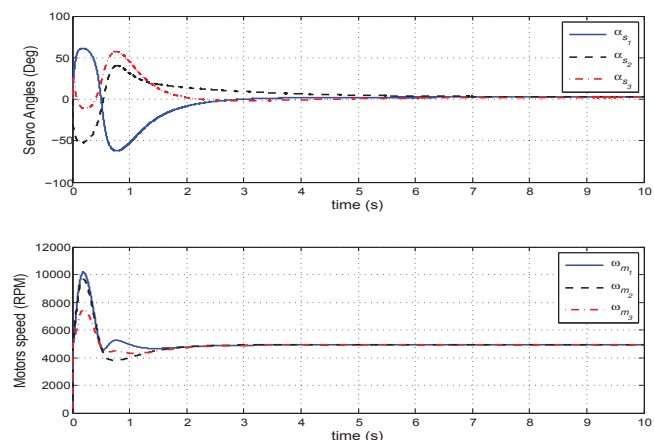

(b) The actuators performance.

Fig. 5. Case 3: Control design for the full system using the two stage feedback linearisation associated with $\mathscr{H}_{\infty}$ LSDP.

of the proposed two stage feedback linearisation technique. The complete model of the tri-rotor UAV including actuators dynamics is:

$$
\begin{aligned}
& \dot{v}=g H_{g}-\boldsymbol{S}(\omega) v+\frac{k_{f}}{M_{t o t}} \boldsymbol{H}_{f} \rho \\
& \dot{\omega}=-\boldsymbol{I}^{-1} \boldsymbol{S}(\omega) \boldsymbol{I} \omega+\boldsymbol{I}^{-1}\left(k_{f} \boldsymbol{H}_{t}-k_{t} \boldsymbol{H}_{f}\right) \rho \\
& \dot{\eta}=\boldsymbol{\Psi} \omega \\
& \dot{\lambda}=\boldsymbol{R}_{b}^{e} v \\
& \dot{x}_{a}=\boldsymbol{A}_{a} x_{a}+\boldsymbol{B}_{a} u_{a} \\
& y_{a}=x_{a} .
\end{aligned}
$$

The input to the system is $u_{a}$ and implementing inputoutput feedback linearisation gives the following feedback linearisation law:

$$
u_{a}=\boldsymbol{\beta}_{f}^{-1}\left(\vartheta_{f}-C_{1} \rho-C_{2}\right),
$$

where

$$
\begin{gathered}
\boldsymbol{C}_{1}=\left[\begin{array}{c}
\left(2 \dot{\boldsymbol{\Psi}} \boldsymbol{I}^{-1}-\boldsymbol{\Psi} \boldsymbol{I}^{-1} \boldsymbol{S}(\omega)+\boldsymbol{\Psi} \boldsymbol{I}^{-1} \boldsymbol{S}(\boldsymbol{I} \omega) \boldsymbol{I}^{-1}\right)\left(k_{f} \boldsymbol{H}_{t}-k_{t} \boldsymbol{H}_{f}\right) \\
\frac{k_{f}}{M_{t o t}} \boldsymbol{R}_{b}^{e} \boldsymbol{S}(\omega) \boldsymbol{H}_{f}
\end{array}\right], \\
C_{2}=\left[\begin{array}{c}
\left(\ddot{\boldsymbol{\Psi}}-2 \dot{\boldsymbol{\Psi}} \boldsymbol{I}^{-1} \boldsymbol{S}(\omega) \boldsymbol{I}-\boldsymbol{\Psi} \boldsymbol{I}^{-1} \boldsymbol{S}(\boldsymbol{I} \omega) \boldsymbol{I}^{-1} \boldsymbol{S}(\omega) \boldsymbol{I}\right) \omega \\
g \boldsymbol{R}_{b}^{e} \boldsymbol{S}(\omega) H_{g}
\end{array}\right]+ \\
{\left[\begin{array}{c}
\left(\boldsymbol{\Psi} \boldsymbol{I}^{-1} \boldsymbol{S}(\omega) \boldsymbol{S}(\omega) \boldsymbol{I}\right) \omega \\
g \boldsymbol{R}_{b}^{e} \boldsymbol{H}_{d g} \boldsymbol{\Psi} \omega
\end{array}\right]+\left[\begin{array}{c}
\boldsymbol{\Psi} \boldsymbol{I}^{-1}\left(\begin{array}{c}
\left.k_{f} \boldsymbol{H}_{t}-k_{t} \boldsymbol{H}_{f}\right) \\
\frac{k_{f}}{M_{t o t}} \boldsymbol{R}_{b}^{e} \boldsymbol{H}_{f}
\end{array}\right] \boldsymbol{N} \boldsymbol{A}_{a} x_{a},
\end{array}\right.}
\end{gathered}
$$


and

$$
\begin{aligned}
& \boldsymbol{N}=\left[\begin{array}{cc}
\operatorname{diag}\left(2 \omega_{m_{i}} \sin \alpha_{s_{i}}\right) & \operatorname{diag}\left(\omega_{m_{i}}^{2} \cos \alpha_{s_{i}}\right) \\
\operatorname{diag}\left(2 \omega_{m_{i}} \cos \alpha_{s_{i}}\right) & \operatorname{diag}\left(-\omega_{m_{i}}^{2} \sin \alpha_{s_{i}}\right)
\end{array}\right]_{6 \times 6}, i=1,2,3 \\
& \boldsymbol{H}_{d g}=\left[\begin{array}{ccc}
\cos \left(\theta_{v}\right) & 0 & 0 \\
\sin \left(\theta_{v}\right) \sin \left(\theta_{v}\right) & -\cos \left(\phi_{v}\right) \cos \left(\theta_{v}\right) & 0 \\
\cos \left(\theta_{v}\right) \sin \left(\theta_{v}\right) & \sin \left(\phi_{v}\right) \cos \left(\theta_{v}\right) & 0
\end{array}\right]
\end{aligned}
$$

The decoupling matrix is defined as:

$$
\boldsymbol{\beta}_{f}=\left[\begin{array}{c}
\boldsymbol{\Psi} \boldsymbol{I}^{-1}\left(k_{f} \boldsymbol{H}_{t}-k_{t} \boldsymbol{H}_{f}\right) \\
\frac{k_{f}}{M_{t o t}} \boldsymbol{R}_{b}^{e} \boldsymbol{H}_{f}
\end{array}\right] \boldsymbol{N} \boldsymbol{B}_{a} .
$$

and its determinant is $\operatorname{det}\left[\boldsymbol{\beta}_{f}().\right]=-k_{d} \omega_{m_{1}}^{3} \omega_{m_{2}}^{3} \omega_{m_{3}}^{3} / \cos \left(\theta_{v}\right)$ where $k_{d}$ is a positive constant related to the specifications of the vehicle. This means that $\boldsymbol{\beta}_{f}($.$) is invertible and the$ feedback linearisation law exists as long as the BLDC motors are operating and the $\cos \left(\theta_{v}\right) \neq 0$. Therefore, the motors should be switched on and operate at low speeds before the controller takes an action to avoid any mathematical error during the initial start of the vehicle. The new control input $\vartheta_{f}$ is defined as $\vartheta_{f}=y^{(3)}$ and the linearised system is a chain of triple integrators with no internal dynamics. The $\mathscr{H}_{\infty}$ LSDP is now invoked to synthesize a controller for the full linearised system using the standard weight design specification of high gain at low frequency and low gain at high frequency in addition to reasonable bandwidth.

Performance of the UAV system and the actuators efforts of this case are shown in Figures 6(a) and 6(b) respectively.

Comparing the decoupling matrices and the feedback linearisation law in both cases, Case 3 (as in (34)) and Case 4 (as in (49)), highlights the strengths of the proposed two stage feedback linearisation technique. The feedback linearisation law resulting from the proposed method is exists for all values of the system states due to the fact that the decoupling matrix $\boldsymbol{\beta}$ in (35) is always nonsingular. In contrary, the decoupling matrix $\boldsymbol{\beta}_{f}$ in (54) is singular when any of BLDC motor has zero speed or when the roll angle $\theta_{v}$ of the vehicle is close to $\pi / 2$. This means that the controller of the two stage feedback linearisation can work for all positions and attitudes of the vehicle and all speeds and angles of the actuators while the feedback linearisation law in Case 4 does not exist for all state values, i.e. when the BLDC motors are not operating singularity exist, and the controller cannot start from static. In terms of performance and actuators efforts, the reader can notice also that the system reaches the steady state value faster when using the two stage feedback linearisation, and this is due to the compensation and control of actuators dynamics in the inner loop.

\section{SUMMARY}

This paper shows the vital importance of actuators dynamics for the control design of nonlinear systems when using dynamic inversion. To implement input-output feedback linearisation while considering actuators dynamics, a two stage feedback linearisation method is developed. The proposed method simplifies the implementation of feedback linearisation when actuators dynamics are considered.
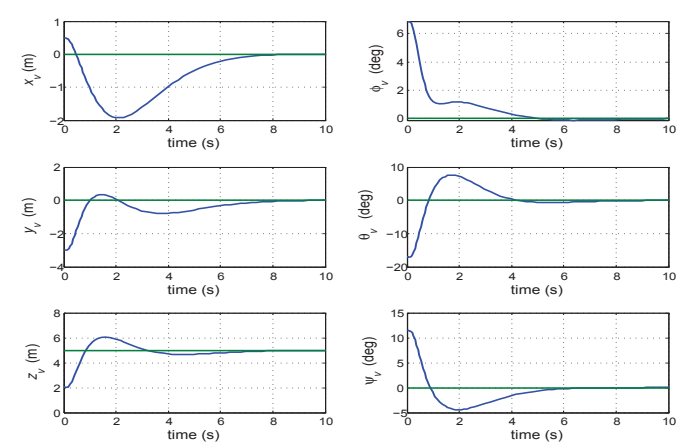

(a) The UAV position and attitude.
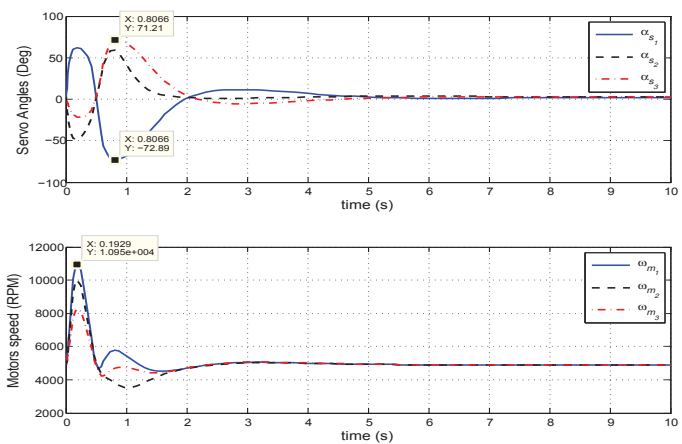

(b) The actuators performance.

Fig. 6. Case4: Control design for the full system using classical feedback linearisation associated with $\mathscr{H}_{\infty}$ LSDP.

\section{REFERENCES}

[1] R. W. Aldhaheri and H. K. Khalil. Effect of unmodeled actuator dynamics on output feedback stabilization of nonlinear systems. $A u$ tomatica, 32(9):1323-1327, 1996.

[2] G. Cai, B. M. Chen, K. Peng, M. Dong, and T. H. Lee. Modeling and control of the yaw channel of a UAV helicopter. IEEE Transactions on Industrial Electronics, 55(9):3426-3434, 2008.

[3] J. Chen and Y. Wang. The guidance and control of small net-recovery UAV. In Proceedings of The 2011 Seventh International Conference on Computational Intelligence and Security (CIS), pages 1566 - 1570, Hainan, China, December 2010.

[4] D. Chwa, J. Y. Choi, and J. H. Seo. Compensation of actuator dynamics in nonlinear missile control. IEEE Transactions on Control Systems Technology, 12(4):620-626, 2004.

[5] A. Isidori. Nonlinear Control Systems. Communications and Control Engineering. Springer, 3 edition, 1995.

[6] Y. Kang and J. K. Hedrick. Linear tracking for a fixed-wing UAV using nonlinear model predictive control. IEEE Transactions on Control Systems Technology, 17(5):1202-1210, 2009.

[7] M. Kara Mohamed. Control and Design of UAV Systems, A Trirotor UAV Case Study. Phd thesis, School of Electrical and Elctronic Engineering, The Univeristy of Manchester, UK, 2012.

[8] M. Kara Mohamed and A. Lanzon. Design and control of novel tri-rotor UAV. In Proceedings of The 2012 UKACC International Conference on Control, pages 304-309, Cardiff, UK, September 2012.

[9] H. A. Khalil. Nonlinear Systems. Prentice Hall, Inc., 2 edition, 2001.

[10] M. Krstić, I. Kanellakopoulos, and P. Kokotović. Nonlinear and Adaptive Control Design. Adaptive and Learning Systems for Signal Processing, Communications, and Control. Wiley-Blackwell, 1995.

[11] B. C. Kuo. Automatic Control Systems. Wiley \& Sons Inc., 7 edition, 1995.

[12] M. S. Mahmoud and M. Zribi. Robust output feedback control for nonlinear systems including actuators. Systems Analysis Modelling Simulation, 43(6):771-792, 2003. 